\title{
EL ROL DEL OCIO EN LA CALIDAD DE VIDA DE LOS ADULTOS MAYORES DE LA PROVINCIA DEL GUAYAS, ECUADOR'
}

\section{LEISURE AND QUALITY OF LIFE OF THE ADULTS BIGGER THAN THE COUNTY OF THE GUAYAS, ECUADOR}

\author{
Miguel A. Bustamante U., ${ }^{1}$ María del C. Lapo M., ${ }^{2}$ Michelle G. Tello S, ${ }^{3}$ Laura Vera S., ${ }^{2}$ \\ Artículo de investigación; proyecto: "Análisis comparativo de la calidad de vida de los adultos mayores de Chile y Ecuador"; fecha de inicio: 4 de marzo de 2015; fecha término: 25 de noviembre de 2016.
}

1 Interdisciplinary Center on Aging, Thematic Task Force on Healthy Aging, CUECH Research Network, Universidad de Talca, Chile.

2 Facultad de Ciencias Económicas y Administrativas de la Universidad Católica de Santiago de Guayaquil.

${ }^{3}$ Asistente de investigación de la Carrera de Economía de la Universidad Católica de Santiago de Guayaquil.

\section{RESUMEN}

Este trabajo analiza la relación que tienen las variables demográficas, situación económica actual y durante la niñez, satisfacción general con la vida y ocio con la calidad de vida y felicidad de los adultos mayores de la provincia del Guayas, Ecuador. Se entrevistó adultos mayores de ambos sexos, de 60 años o más que residen en zonas urbanas y rurales de la provincia de Guayas, Ecuador y se planteó un modelo logit ordinal de ocio y familia sobre satisfacción con la vida. Resultando que la familia es fuente de felicidad y tienen una mayor probabilidad de satisfacción con la vida y que el ocio difiere según nivel de educación, residencia, estado civil, situación económica y satisfacción general con la vida.

Palabras clave: Adultos mayores, ocio, calidad de vida, felicidad subjetiva, satisfacción con la vida.
This work analyzes the relationship that you/they have the demographic variables, current economic situation and during the childhood, general satisfaction with the life and leisure with the quality of life and the adults' happiness bigger than the county of the Guayas, Ecuador. She/he interviewed adults bigger than both sexes, 6o years old or more than they reside in urban and rural areas of the county of Guayas, Ecuador and she/he thought about a model ordinal logit of leisure and family has more than enough satisfaction with the life. Being that the family is source of happiness and they have a bigger probability of satisfaction with the life and that the leisure differs according to education level, residence, civil state, economic situation and general satisfaction with the life.

Keywords: Elderly, leisure, quality oflife, subjective happiness, life satisfaction. 


\section{Introducción}

La población mundial está envejeciendo de forma acelerada estimándose que entre el año 2015 y 2050 la proporción de los habitantes del planeta mayores a 60 años se duplicará, pasando del $12 \%$ al $22 \%$; con una esperanza de vida superior o igual a los 60 años (OMS, 2016). Por otra parte, en Ecuador se prevé que para el año 2025 la población de 65 años y más se incrementará del $6 \%$ al $9 \%$ como resultado de los cambios en las tasas de mortalidad y fecundidad (INEC, 2016), en consecuencia, si las personas viven más años y estos años adicionales se traducen en una disminución de la capacidad física y mental, las consecuencias para la Calidad de Vida (CV) de la sociedad serían negativas (OMS, 2015).

\section{Calidad de vida}

La Calidad de Vida es un constructo multidimensional que comprende un conjunto de condiciones que cada persona necesita para llevar a cabo su propio proyecto personal en condiciones de integridad humana. La $\mathrm{CV}$ se define como la percepción del individuo en su posición en la vida en el contexto de su cultura y valores y en relación con sus objetivos, expectativas, estándares e intereses (OMS, 2015), por consiguiente, la CV es el resultado de la evaluación que cada persona realiza de los elementos objetivos y subjetivos de las distintas dimensiones que conforman su vida, sujeto a la etapa evolutiva en la que se encuentra la persona (Urzúa, Loyola, Navarrete y Valenzuela, 2014).

La CV es entonces la valoración que cada individuo hace de lo que considera bueno para sí mismo e incluye sus valores, preferencias y deseos dando forma a diversos componentes tales como una adecuada salud física y psicológica; un status socioeconómico y laboral que le permita satisfacer sus necesidades básicas; la posibilidad de acceso a los bienes y servicios de la sociedad a la que pertenece; alcanzar el reconocimiento de la sociedad acerca de sus derechos, valores y cualidades para mantener un nivel adecuado de autoestima; un medio ambiente sano y libre de contaminantes; y valorar el sentido de pertenencia a una sociedad justa que le permita el libre acceso a sus derechos y libertades (Herrera y Guzmán, 2012).

Para medir la CV usualmente se ha recurrido a las circunstancias o dimensiones objetivas de la vida a través de indicadores sociales. También a la valoración que hacen las personas de las experiencias o bienestar subjetivo de la vida que considera las reacciones emocionales de las personas, la satisfacción con dominios específicos de la vida y la satisfacción con la vida misma como un todo, incluyendo un componente cognitivo y otro afectivo de valoración (Brajša-Žganec, Merkaš y Šverko, 2010; Diener, Suh, Lucas y Smith, 1999; Diener y Suh, 1997).

El componente cognitivo de la CV subjetiva se expresa como la satisfacción vital, ya sea de manera global o por dominios específicos, y el componente afectivo se refiere a la presencia de sentimientos positivos o también llamado felicidad (Moyano y Ramos, 2007). La satisfacción con la vida es generalmente conceptualizada como la sensación individual de bienestar que se manifiesta por la satisfacción o insatisfacción con las áreas o dominios que son importantes para el individuo, tales como el estado físico y sicológico, grado de independencia o funcionalidad, así como las relaciones más destacadas de su entorno (Michalkó y Rátz, 2010). Estudios previos han identificado variables que influyen en la satisfacción con la vida, por ejemplo el nivel socio económico, disponibilidad de recursos económicos, satisfacción con la alimentación, percepción de estado de salud, discrepancia del yo, edad, género, edad, estatus marital, tamaño de la familia y nivel educativo (Schnettler, Miranda, Sepúlveda, Orellana, Denegri, Mora y Lobos, 2014).

En un sentido amplio el envejecimiento saludable está compuesto por un conjunto de elementos como la satisfacción con la vida, la salud física y mental y la CV (Heo, Stebbins, Kim. y Lee, 2013). Por otra parte, sobre la base de las estimaciones de la progresiva longevidad de la población mundial, el tiempo de ocio parece jugar un papel cada vez más importante en la vida de las personas, quienes, junto con la familia, el trabajo o las amistades (Aristegui. y Silvestre, 2012) explican parte importante de la CV; una vez que éstas satisfacen sus necesidades biológicas básicas y, psicológicas tales como el tiempo de ocio que adquiere mayor relevancia y ocupa un lugar importante como fuente del bienestar subjetivo del individuo (Diener, Suh, Lucas. y Smith, 1999).

Las definiciones de ocio son diversas. Algunas lo definen como el tiempo discrecional remanente después del trabajo (Newman, Tay y Diener, 2013); otros, como una actividad libremente elegida, diversión u ocupación reposada o como un descanso o cambio de otras tareas cotidianas (Narro, Aleixandre y Meléndez, 1994). Por otra parte, el ocio en un concepto más moderno se identifica con el descanso, las vacaciones, el espectáculo y la diversión, sin embargo, las teorías que se han desarrollado desde inicios de este siglo lo consideran como una experiencia humana que proporciona autorrealización y calidad de vida, por cuanto se trata de una experiencia elegida o libremente deseada (Cuenca, 2007).

En el caso de los adultos mayores (AM) el ocio puede llegar a ser más importante después de la disminución del tiempo dedicado a las actividades laborales (Griffin. y McKenna, 1999; Hersch, 1991). Varios estudios acerca de la relación existente entre el ocio y la satisfacción con la vida se encuentran descritos en la literatura, por ejemplo Havighurst y Albrecht (1953) lo definen como el tiempo libre disponible después del retiro laboral, mismo que provee la oportunidad para mantener altos niveles de actividad que son esenciales para la satisfacción y enriquecimiento personal (Lefrancois, Leclerc y Poulin, 1997). Por una parte, porque los AM tienden a reemplazar los roles perdidos con actividades nuevas para mantener los niveles de actividad (Griffin y McKenna, 1999; Lemon, Bengtson y Peterson, 1972; Leung. y Lee, 2005; Rodríguez, Látková y Sun, 2007) o, por otra parte, porque mientras mayores sean los niveles de participación en actividades de ocio, serán superiores los niveles de bienestar subjetivo. Así mismo, Wendel-Vos, Schuit, Tijhuis y Kromhout (2004) documentaron relaciones positivas directas entre la participación en actividades físicas inherentes al ocio y calidad de vida relacionada con la salud. Lloyd y Auld (2002) encontraron relaciones positivas directas entre las actividades sociales, tales como visitar familiares o salir con amigos, con el bienestar subjetivo. Finalmente Robinson y Martin (2008) mostraron que las personas que son felices participan en más actividades sociales. 
Los hallazgos anteriormente descritos son consistentes con la Teoría de las Necesidades de Maslow (1991), la cual postula que la satisfacción de las necesidades tiene efectos positivos en el bienestar subjetivo por cuanto si las personas satisfacen sus necesidades podrán mantener e incrementar su satisfacción vital (Diener y Lucas, 200o). En esta misma línea, Tinsley y Eldredge (1995) confirmaron que el ocio tiene un efecto beneficioso en el bienestar subjetivo y la salud de las personas por cuanto satisfacen sus necesidades de recreación y esparcimiento. Complementariamente, la Teoría de la Gero-trascendencia, desarrollada por Tornstam (2003), señala que la etapa del envejecimiento presenta la oportunidad para madurar y obtener sabiduría a través del desarrollo trascendental, como es la redefinición del ser hacia una perspectiva superior, normalmente acompañada por un aumento en la satisfacción por la vida. En sentido contrario, la Teoría del Retraimiento, desarrollada por Cumming y Henry (1961), plantea que es natural e inevitable para los adultos mayores retirarse de las actividades productivas a medida que van envejeciendo, desvinculándose de la sociedad a la que pertenece, la misma que tiende a rechazarlo por cuanto el adulto mayor no consigue adaptarse a la sociedad en la que vive. Esta teoría supone que los adultos mayores deben desvincularse de la sociedad en la que viven ya que esta desvinculación es un proceso innato $\mathrm{y}$ funcional, tanto para el adulto mayor como para la sociedad y que prepara a ambos para la muerte (Alcalde y Laspeñas, 2005), generando en consecuencia un impacto negativo en la satisfacción con la vida (Parker, 1996).

La literatura reconoce a Havighurst (1961) como el pionero en utilizar el concepto de envejecimiento activo como un proceso exitoso en términos de felicidad y satisfacción vital, de forma tal que los adultos mayores que envejecen y hacen un uso positivo del ocio, logran mayor éxito, están satisfechos, son activos, independientes y autosuficientes en sus vidas. Es así como, se ha demostrado que el ocio juega un papel importante en la satisfacción con la vida de las personas en general y de los adultos mayores en particular (Fernández-Ballesteros, Zamarrón y Ruíz, 2001; Griffin y McKenna, 1999; Newman, Munné y Codina, 1996; Nimrod, 2007; Tay y Diener, 2013), confirmándose que aquellos adultos mayores que incrementan su participación en diferentes actividades tienden a percibir una mejora en su satisfacción general con la vida. Esto porque las actividades recreativas o de ocio activo, proveen un mecanismo para hacer frente al tiempo libre que experimentan quienes ya no forman parte de la fuerza laboral (Griffin y McKenna, 1999). En consecuencia, los adultos mayores que han llegado a esa etapa de su vida según su propia historia, particularidades y la forma en que han afrontado sus experiencias y aprendizajes en las instancias previas de su desarrollo, valoran de manera diferente cada aspecto de su vida, considerando más importantes algunas cosas y otras no (Urzúa, Loyola, Navarrete y Valenzuela, 2014).

\section{Metodología}

Se tuvo a la vista los fundamentos conceptuales que definen la calidad de vida y de cómo ésta depende del comportamiento de variables que provienen de diversas dimensiones de la vida (Brajša-Žganec, Merkaš y Šverko, 2010; Diener, Suh, Lucas y Smith, 1999; Diener y Suh, 1997; Griffin. y McKenna, 1999;
Herrera y Guzmán, 2012; Leung y Lee, 2005; WendelVos, Schuit, Tijhuis y Kromhout, 2004; Lemon, Bengtson y Peterson, 1972; Rodríguez, Látková y Sun, 2007; Urzúa, Loyola, Navarrete y Valenzuela, 2014), por lo tanto este trabajo analiza la relación que tienen las variables demográficas, situación económica actual y durante la niñez, respecto de la satisfacción general con la vida y ocio, como componentes de la calidad de vida y felicidad de los adultos mayores del Guayas, Ecuador.

\section{Diseño, muestra y participantes}

Se entrevistó a personas adultos mayores de ambos sexos, de 60 años o más que residen en zonas urbanas y rurales de la provincia de Guayas, Ecuador. Se aplicó muestreo bietápico, estratificado por conglomerados con sub-muestreo incidental (casual) y por redes (bola de nieve) dentro del conglomerado (Eyssautier, 2006; Hernández, Fernández y Baptista, 2006). Como estratos se accedió a los 25 cantones de la provincia de Guayas con afijación proporcional al tamaño de la población muestreada. Dentro de cada cantón se realizó la afijación por conglomerado, también de forma proporcional al tamaño de la población muestreada. El estrato quedó finalmente como la intersección de cantón y conglomerado. Como conglomerados se utilizaron los centros gerontológicos seleccionados mediante un muestreo aleatorio simple, con la función "muestra aleatoria de casos” de SPSS (Pérez López, 2005).

El nivel de error absoluto máximo esperado de los resultados del cuestionario es de $\pm 5 \%$ para un nivel de confianza del 95\% (Walpole, Myers, Myers y Ye, 2007). El cuestionario fue aplicado personalmente durante los meses de marzo a julio 2015 por entrevistadores debidamente entrenados. Así mismo, la muestra fue de 817 adultos mayores residentes en la provincia del Guayas. Del total de los encuestados, 429 fueron hombres y 388 mujeres; la edad promedio reportada fue de 74 años y la edad máxima fue de 101 años.

\section{Escalas y medidas utilizadas}

Se definieron como variables dependientes dos medidas de CV subjetiva. Primero, la satisfacción con la vida (SWLS, Satisfaction with Life Scale) (Diener Emmons, Larsen y Griffin, 1985) que consta de 5 ítems valuados en una escala Likert (Likert, 1932) en 6 niveles (5-30). Segundo, la felicidad subjetiva (SHS, Subjective Happiness Scale) (Lyubomirsky y Lepper, 1999) que cuenta con 5 ítems medidos en una escala Likert de 7 puntos (4-28). Empíricamente ambas medidas, aunque están correlacionadas, han sido utilizadas conjuntamente (Theurer y Wister, 2010), aunque es más útil separarlas debido a que son conceptos que difieren desde el punto de vista de su dimensión temporal.

La información sociodemográfica incluida en el cuestionario fue la edad (en años), género ( $1=$ masculino, 2 = femenino), estatus marital $(1=$ soltero, 2 = separado, $3=$ divorciado, $4=$ viudo, 5 = casado), educación $(1=\sin$ educación, $2=$ con educación primaria o secundaria, $3=$ con educación técnica o universitaria), número de niños viviendo en el hogar y etnia, fueron incluidos en el cuestionario. También se preguntó a los entrevistados sobre la satisfacción con su situación económica actual (SWES, Satisfaction with Economic Situation), en este caso con un ítem y una escala Likert (44) de 6 
puntos (1-6) $(1=$ extremadamente insatisfecho a $6=$ extremadamente satisfecho, escala que recodificamos en tres categorías: $\mathbf{1}=$ insatisfecho, $\mathbf{2}=$ medianamente insatisfecho y 3 = satisfecho).

\section{Datos y análisis estadístico}

Se explicó a los entrevistados los objetivos del cuestionario y se les indicó el carácter estrictamente confidencial del tratamiento de la información obtenida (Hernández, Fernández y Baptista, 2006). Los entrevistados firmaron el consentimiento informado antes de responder el cuestionario. La ejecución de este estudio fue aprobada por el Comité de Bioética de la Universidad Católica de Santiago de Guayaquil (UCSG). Los resultados fueron analizados usando el paquete estadístico SPSS v. 22 para Windows en español. Se realizó estadística descriptiva para describir la muestra, así como para conocer qué porcentaje de la muestra reportaba alto o bajo nivel de acuerdo con respecto a la satisfacción con su vida. Con las variables trabajo, familia, amigos, ocio y la alimentación se realizó un análisis del nivel de significancia.

Las variables fueron recodificadas como binarias (1 = importante fuete de felicidad y o = no importante fuente de felicidad). La fiabilidad de las escalas fue probada con el alfa de Cronbach (1982), además se aplicó prueba Chi-cuadrado para verificar la existencia de diferencias significativas en el grado de satisfacción con la vida de acuerdo a la situación económica, nivel de educación, y nivel de residencia, entre otras variables. Finalmente, se planteó un modelo logit ordinal para contrastar la influencia de las variables ocio y familia sobre la satisfacción con la vida (Eyssautier, 2006), donde la variable dependiente fue SWLS y las variables explicativas fueron ocio y familia.

\section{Resultados}

Los resultados que a continuación se detallan, contienen los hallazgos obtenidos en las diversas fases de la investigación dando cuenta de la ejecución exhaustiva del método previamente descrito.

\section{Análisis demográfico}

Un $52,3 \%$ de la muestra son hombres (Tabla 1), 45,5\% casados y un $31,5 \%$ viudos. En su mayoría $(39,4 \%)$ residen en un pueblo rural, un $33,5 \%$ reside en una gran ciudad y en educación el $14 \%$ se declara sin estudios y la gran mayoría $(53,1 \%)$ cursaron enseñanza básica incompleta y básica completa.

Tabla 1 Demografía de la muestra

\begin{tabular}{|c|c|c|c|}
\hline Género & & Femenino & $47.5 \%$ \\
\hline Su estado civil actual es: & $\%$ & Ud. reside: & $\%$ \\
\hline Soltero & 9.3 & \multirow{5}{*}{$\begin{array}{l}\text { En una gran ciudad } \\
\text { En una pequeña ciudad } \\
\text { En un pueblo rural } \\
\text { En el campo }\end{array}$} & \multirow{5}{*}{$\begin{array}{l}33.5 \\
16.5 \\
39.4 \\
10.5\end{array}$} \\
\hline Separado & 9.2 & & \\
\hline Divorciado & 4.5 & & \\
\hline Viudo & 31.5 & & \\
\hline Casado & 45.5 & & \\
\hline ¿Cuál es el nivel de educación? & \multicolumn{2}{|l|}{$\%$} & $\%$ \\
\hline Sin estudios & 14.0 & Universitaria Completa & 6.7 \\
\hline Básica incompleta & 29.1 & Universitaria Incompleta & 3.8 \\
\hline Básica completa & 24.0 & Técnica Incompleta & 0.4 \\
\hline Bachillerato incompleto & 11.4 & Técnica Completa & 1.1 \\
\hline Bachillerato completo & 9.2 & Tecnológico Completo & 0.4 \\
\hline
\end{tabular}

Como se observa en la Tabla 2, el 38,1\% de los adultos mayores se encuentra bastante o completamente satisfecho y un $61,9 \%$ manifestaron insatisfacción total o parcial con su situación económica actual. Respecto a la situación económica durante su niñez el 39,4\% de los encuestados se consideró muy pobre, el $27,3 \%$ bajo el promedio, el $24,8 \%$ de clase media, $7 \%$ sobre el promedio y $1,5 \%$ rico. Finalmente un $63,3 \%$ de los AM encuestados refirieron aportar el ingreso principal en el hogar. Además, de los resultados de la satisfacción con la vida se observa que el 9,6\% de los adultos mayores están completamente, bastante, o un poco en desacuerdo, en contraste con el 9o,4\% que se encuentran un poco, bastante o completamente de acuerdo, manifestándose satisfechos con su vida.

Tabla 2 Percepción de situación económica en la niñezy en la actualidad

\begin{tabular}{|c|c|c|c|c|c|}
\hline $\begin{array}{l}\text { Respecto a su actual situación } \\
\text { económica, usted en general está: }\end{array}$ & Frecuencia & $\%$ & & Frecuencia & $\%$ \\
\hline Completamente insatisfecho & 8 & 1 & Un poco satisfecho & 347 & 42.5 \\
\hline Bastante insatisfecho & 49 & 6 & $\begin{array}{l}\text { Bastante satisfecho } \\
\text { Completamente }\end{array}$ & 252 & 30.8 \\
\hline Un poco insatisfecho & 101 & 12.4 & satisfecho & 60 & 7.3 \\
\hline Total & & & & 817 & 100 \\
\hline
\end{tabular}




\begin{tabular}{|c|c|c|c|c|c|c|}
\hline $\begin{array}{l}\text { Respecto a su situación económica } \\
\text { durante la niñez, usted fue: }\end{array}$ & \multicolumn{2}{|r|}{ Frecuencia } & $\%$ & & Frecuencia & $\%$ \\
\hline \multirow{3}{*}{$\begin{array}{l}\text { Muy pobre } \\
\text { Bajo el promedio }\end{array}$} & \multicolumn{2}{|r|}{322} & 39.4 & \multirow{3}{*}{$\begin{array}{l}\text { Clase media } \\
\text { Sobre el promedio } \\
\text { Rico }\end{array}$} & 203 & 24.8 \\
\hline & \multirow{2}{*}{\multicolumn{2}{|c|}{223}} & 27.3 & & 57 & 7 \\
\hline & & & & & 12 & 1.5 \\
\hline Total & & & & & 817 & 100 \\
\hline \multirow{2}{*}{$\begin{array}{l}\text { ¿Es Ud. la persona que aporta el } \\
\text { ingreso principal en su hogar? }\end{array}$} & \multirow{2}{*}{$\mathrm{Si}$} & \multicolumn{2}{|c|}{ Frecuencia } & 517 & Frecuencia & 300 \\
\hline & & \multicolumn{2}{|l|}{$\%$} & 63.3 & $\%$ & 36.7 \\
\hline ¿Está satisfecho con su vida? & & Frecuencia & $\%$ & & Frecuencia & $\%$ \\
\hline En desacuerdo: completamente & & 3 & 0.4 & De acuerdo: un poco & 247 & 30.2 \\
\hline En desacuerdo: bastante & & 21 & 2.6 & $\begin{array}{l}\text { De acuerdo: bastante } \\
\text { De acuerdo: }\end{array}$ & 344 & 42.1 \\
\hline En desacuerdo : un poco & & 54 & 6.6 & completamente & 148 & 18.1 \\
\hline Total & & & & & 817 & 100 \\
\hline ¿Usted está en general? & & Frecuencia & $\%$ & & Frecuencia & $\%$ \\
\hline Completamente insatisfecho & & 2 & 0.2 & Un poco satisfecho & 316 & 38.7 \\
\hline Bastante insatisfecho & & 20 & 2.4 & $\begin{array}{l}\text { Bastante satisfecho } \\
\text { Completamente }\end{array}$ & 361 & 44.2 \\
\hline Un poco insatisfecho & & 38 & 4.7 & satisfecho & 80 & 9.8 \\
\hline Total & & & & & 817 & 100 \\
\hline
\end{tabular}

Esta percepción se ratifica en la percepción general con el $54 \%$ de los AM que manifiestan sentirse bastante o completamente satisfechos. Un $43,4 \%$ se encuentran poco satisfechos o insatisfechos y sólo el 2,6\% manifestaron bastante o completa insatisfacción.

Los resultados del análisis de la felicidad se obtiene que la fuente de felicidad más importante para los adultos mayores es la familia, con una media de 4,61 (D.S. $=0,741)$, seguida de la alimentación $(4,46)$, los amigos $(4,15)$, el trabajo $(3,93)$, dejando el últimolugar para el ocio con una media de 3,73 (D.S. $=1,090)$. Sin embargo, los adultos mayores, al ser entrevistados respecto del ocio en relación a la felicidad, un $64 \%$ respondió estar de acuerdo o muy de acuerdo con el ocio como fuente de felicidad.

Satisfacción con la vida y características sociodemográficas

De la Tabla 3 se observa que el género $(\mathrm{p}<0,05)$, la educación $(\mathrm{p}<0,05)$ y la satisfacción general con la vida $(0,01)$ explican el grado de acuerdo o desacuerdo con el ocio como fuente de felicidad. Por otra parte, no resultaron estadísticamente significativos el lugar de residencia, el estado civil, la situación económica en la niñez y si el adulto mayor es el responsable principal de los ingresos de su hogar.

Tabla 3 Satisfacción con la vida y demografía de la muestra

\begin{tabular}{|c|c|c|c|c|c|c|c|c|c|c|c|}
\hline \multirow{2}{*}{\multicolumn{5}{|c|}{$\begin{array}{l}\text { Grado de acuerdo con el ocio como fuente de felicidad } \\
\text { Diferencias entre muestras }\end{array}$}} & \multicolumn{2}{|c|}{ En desacuerdo } & \multicolumn{4}{|c|}{ De acuerdo } & \multirow[t]{2}{*}{ Nivelp } \\
\hline & & & & & \multirow[t]{2}{*}{$\mathrm{n}$} & \multicolumn{2}{|l|}{$\%$} & \multirow[t]{2}{*}{$\mathrm{n}$} & \multicolumn{2}{|c|}{$\%$} & \\
\hline \multicolumn{5}{|l|}{ Género } & & & & & & & 0,045 \\
\hline \multirow{2}{*}{\multicolumn{5}{|c|}{$\begin{array}{l}\text { Masculino } \\
\text { Femenino }\end{array}$}} & 166 & \multicolumn{2}{|l|}{$38,70 \%$} & 166 & \multicolumn{2}{|c|}{$61,30 \%$} & \\
\hline & & & & & 124 & \multicolumn{2}{|l|}{$32,00 \%$} & 124 & \multicolumn{2}{|c|}{$68,00 \%$} & \\
\hline \multicolumn{5}{|l|}{ Lugar de residencia } & & & & & & & 0,367 \\
\hline \multirow{2}{*}{\multicolumn{5}{|c|}{$\begin{array}{l}\text { Rural } \\
\text { Urbano }\end{array}$}} & 139 & \multirow{2}{*}{\multicolumn{2}{|c|}{$\begin{array}{l}47,93 \% \\
52,07 \%\end{array}$}} & & \multirow{2}{*}{\multicolumn{2}{|c|}{$\begin{array}{l}51,23 \% \\
48,77 \% \\
\end{array}$}} & \\
\hline & & & & & 151 & & & & & & \\
\hline \multirow[t]{2}{*}{ Nivel de educación } & $\mathrm{n}$ & $\%$ & $\mathrm{n}$ & $\%$ & \multicolumn{2}{|c|}{ Nivel de educación } & $\mathrm{n}$ & $\%$ & $\mathrm{n}$ & $\%$ & 0,000 \\
\hline & & & & & \multicolumn{2}{|c|}{ Universidad } & & 1.72 & & & \\
\hline Sin estudios & 48 & $16,55 \%$ & 66 & $12,52 \%$ & \multicolumn{2}{|c|}{$\begin{array}{l}\text { Incompleta } \\
\text { universidad }\end{array}$} & 18 & $\begin{array}{c}\% \\
6,21\end{array}$ & 37 & $4,93 \%$ & \\
\hline Básica incompleta & 100 & $34,48 \%$ & 138 & $26,19 \%$ & \multicolumn{2}{|c|}{ Completa } & 5 & $\begin{array}{c}\% \\
0,69\end{array}$ & 26 & $7,02 \%$ & \\
\hline \multirow{3}{*}{$\begin{array}{l}\text { Básica completa } \\
\text { Bachillerato } \\
\text { incompleto } \\
\text { Bachillerato } \\
\text { completo }\end{array}$} & 72 & $24,83 \%$ & 124 & $23,53 \%$ & \multicolumn{2}{|c|}{$\begin{array}{l}\text { Técnica } \\
\text { incompleta }\end{array}$} & 2 & $\%$ & 1 & $0,19 \%$ & \\
\hline & 20 & $6,90 \%$ & 73 & $13,85 \%$ & \multicolumn{2}{|c|}{$\begin{array}{l}\text { Técnica completa } \\
\text { Tecnológico }\end{array}$} & 3 & $\begin{array}{c}\% \\
0,34\end{array}$ & 6 & $1,14 \%$ & \\
\hline & 21 & $7,24 \%$ & 54 & $10,25 \%$ & Com & & 1 & $\%$ & 2 & $0,38 \%$ & \\
\hline
\end{tabular}




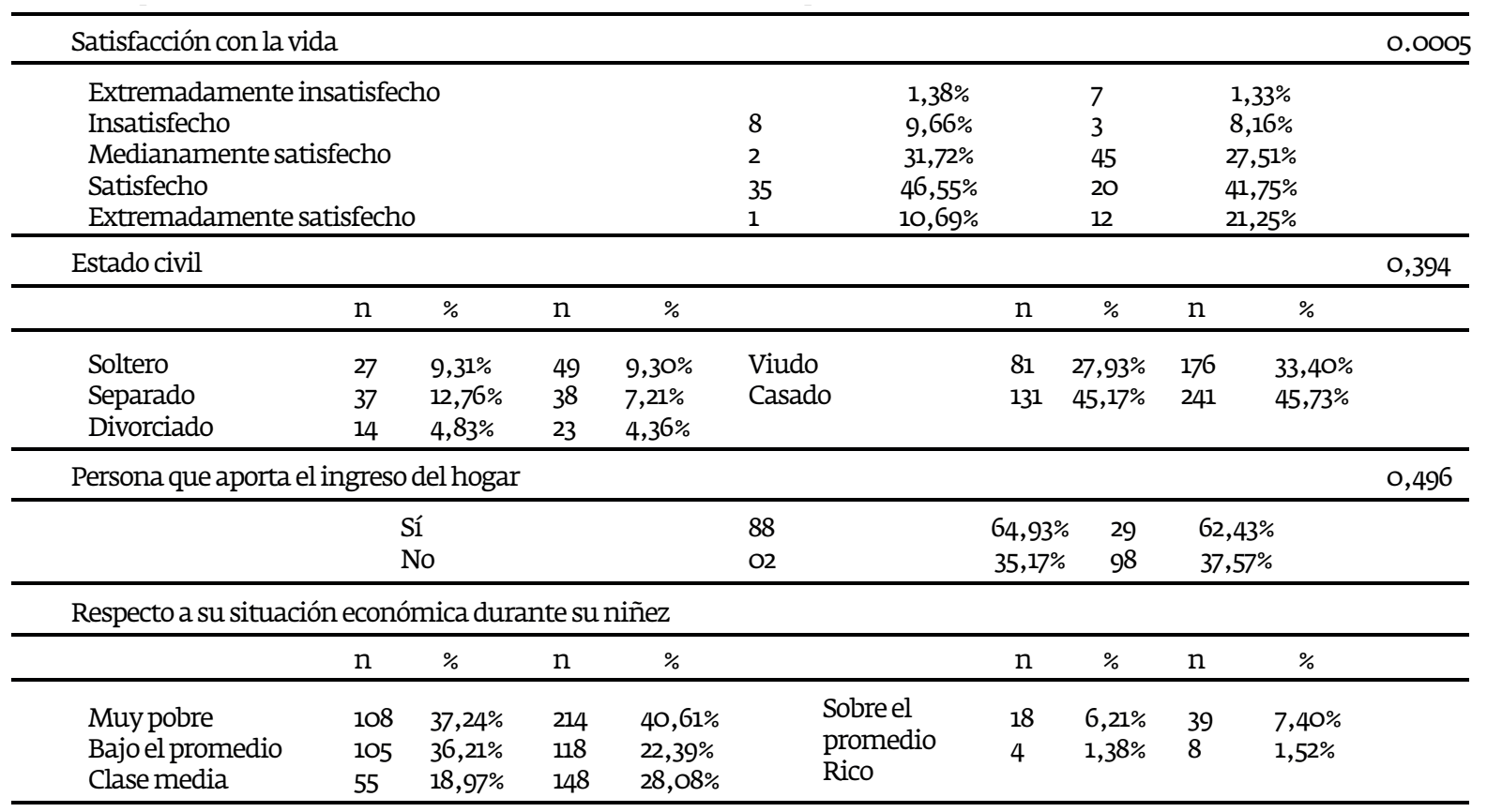

Se destaca la significancia $(\mathrm{P}<0,05)$ de personas que considera al ocio como fuente de felicidad en ambos géneros: masculino $(61,3 \%)$ y femenino $(68 \%)$. Además en la muestra se observa un $23.5 \%$ significativo ( $\mathrm{p}<$ $\mathrm{o}, 01$ ) de $A M$ con educación básica completa que aprecia y que no aprecia $(24,8 \%)$ al ocio como fuente de felicidad. Se destacan también las altas proporciones en el nivel de educación básica incompleta donde el porcentaje de AM que está en desacuerdo con el reactivo $(34.5 \%)$ fue la mayor en toda la muestra. Así mismo, resalta las significativas altas proporciones $(\mathrm{p}<1 \%)$ de AM medianamente satisfechos $(31.7 \%)$ y no satisfechos (46.6\%) con su vida en general, pero que no consideran al ocio como fuente de felicidad.
Por último, de la Tabla 4 se observa que el modelo ordinal desarrollado con $\left(^{*}\right) \mathrm{p}$ valor $\leq 1 \%$, muestra resultados diversos. Por una parte, se determina que la probabilidad de una alta satisfacción con la vida disminuye en la medida en que los adultos mayores no consideren importante la familia como fuente de felicidad $(\beta=-0,95)$. Además, la probabilidad de alta satisfacción con la vida aumenta cuando la persona no considera importante el ocio como fuente de felicidad $(\beta=0,447)$.

Tabla 4 Resultado modelo logit ordinal

\begin{tabular}{lllll}
\hline Variable dependiente & $\mathrm{p}=0,000$ & \multicolumn{4}{c}{ Satisfacción con la vida SWLS } \\
\hline Variables explicativas & $\mathrm{B}$ & Error Estand. & $\mathrm{P}$ \\
\hline Importancia de la familia $=0$ & $-0,95^{*}$ & $\mathrm{0}, 303$ & 0,002 \\
Importancia de la familia $=1$ & - & - & - \\
Importancia del ocio $=0$ & $0,447^{*}$ & 0,13 & 0,001 \\
Importancia del ocio $=1$ & - & - & - \\
\hline
\end{tabular}

\section{Discusión}

Más del 50\% de la muestra reporta estar satisfecho con respecto a su vida y con su vida en general, ratificando el hecho que la CV es una evaluación general que realiza la persona acerca de sí misma a partir de diversas dimensiones (Urzúa, Loyola, Navarrete y Valenzuela, 2014) objetivas y subjetivas que conforman su vida. En consecuencia, la $\mathrm{CV}$ es efectivamente una valoración personal que el individuo hace de lo que considera bueno para sí (Herrera y Guzmán, 2012) y que de forma multidimensional satisfacen las diversas circunstancias, experiencias y dimensiones de bienestar objetivo y subjetivo que, a lo largo de su vida, ha sabido enfrentar y sobrellevar (Diener, Suh, Lucas y Smith, 1999; Diener y Suh, 1997; BrajšaŽganec, Merkaš y Šverko, 2010).
Los adultos mayores, al evaluar los diversos dominios de la vida (Heo, Stebbins, Kim y Lee, 2013; Schnettler, Miranda, Sepúlveda, Orellana, Denegri, Mora y Lobos, 2014) evalúan positivamente a la familia asignándole un puntaje promedio de 4,6 en una escala de 5 puntos, valorándola como principal fuente de felicidad lo que les permite expresar un componente de afectividad relevante (Aristegui y Silvestre, 2012; Moyano y Ramos, 2007) respecto de las demás dimensiones.

La consideración del ocio (Cuenca, 2007; Newman, Tay y Diener, 2013) como fuente de felicidad (Heo, Stebbins, Kim y Lee, 2013; Schnettler, Miranda, Sepúlveda, Orellana, Denegri, Mora y Lobos, 2014) difiere de acuerdo con el nivel de educación, lugar de residencia, estado civil, situación económica durante 
la niñez, situación económica actual y con respecto a la satisfacción general con la vida. Sin embargo, un $64 \%$ respondió estar de acuerdo o muy de acuerdo con el ocio como fuente de felicidad. Estos resultados ratifican la importancia del ocio en la vida humana (Griffin y McKenna, 1999; Hersch, 1991) mismos que han sido muy bien explicados en la literatura (Havighurst y Albrecht, 1953).

Se ratifica que las distinciones de género (Walpole, Myers, Myers, y Ye, 2007), educación y satisfacción general con la vida explican de forma significativa el grado de acuerdo o desacuerdo con el ocio como fuente de felicidad, sea en beneficio del enriquecimiento personal que les depara el ocio (Lefrancois, Leclerc y Poulin, 1997) así como para reponer o sustituir los roles perdidos con nuevas actividades factibles ahora por el ocio que enfrentan (Griffin y McKenna, 1999; Lemon, Bengtson. y Peterson, 1972; Leung y Lee, 2005; Rodríguez, Látková y Sun, 2007; WendelVos, Schuit, Tijhuis y Kromhout, 2004) lo que les permita satisfacer sus necesidades sus necesidades de recreación y esparcimiento (Maslow, 1991; Tinsley y Eldredge, 1995), en camino hacia una madurez superior y obtención de sabiduría a través del desarrollo trascendental (Tornstam, 2003).

En general los AM muestran conductas en contrario de la Teoría del Retraimiento desarrollada por Cumming y Henry (1961), quien consideraba natural e inevitable retirarse de las actividades productivas a medida que se envejece, considerando este proceso como necesario y funcional para los adultos mayores como para la sociedad (Alcalde y Laspeñas, 2005). Esto debido a que persistir en mantenerse en actividad produciría impactos negativos en la satisfacción con la vida (Parker, 1996); confirmando a través de sus apreciaciones que aquellos adultos mayores que incrementan su participación en diferentes actividades de trabajo, esparcimiento vinculación social y pertenencia a los grupos familiares y de amigos, tienden a percibir una mejora sustancial en su satisfacción general con la vida (FernándezBallesteros, Zamarrón y Ruíz, 2001; Griffin y McKenna, 1999; Munné. y Codina, 1996; Newman, Tay y Diener, 2013; Nimrod, 2007).

Por otra parte y también confirmado estadísticamente (Walpole, Myers, Myers y Ye, 2007), no resultaron significativos para los AM ellugar de residencia, estado civil, situación económica en la niñez y si el adulto mayor es el responsable principal de los ingresos de su hogar, lo que permite afirmar que los AM, que llegan a esta etapa de sus vidas, sea mostrando conductas de envejecimiento activo (Havighurst, 1961), recorrido su propia historia, enfrentado sus experiencias y aprendizajes materializando el concepto de gerotrascendencia de Tornstam (2003), evalúan de manera diferencial los diversos aspecto de la vida, valorando más importantes algunas cosas y otras no (Urzúa, Loyola, Navarrete y Valenzuela, 2014).

Los AM que consideran a la familia (Aristegui y Silvestre, 2012) como fuente de felicidad tienen una mayor probabilidad de satisfacción con la vida; sin embargo, las personas que están de acuerdo sobre el hecho de que el ocio (Newman, Tay y Diener, 2013) es una fuente de felicidad, tienen menor probabilidad de satisfacción con la vida. Ello parece concordante con el hecho que resultan significativas las proporciones de AM medianamente satisfechos y satisfechos con su vida en general pero que no consideran al ocio como fuente de felicidad.

\section{Conclusiones}

De los datos analizados y realizados los estudios pertinentes se concluye que más de la mitad de los AM entrevistados reportan satisfacción con su vida valorando muy positivamente la familia como fuente de felicidad. Seguidamente los AM aprecian el ocio como fuente de felicidad, sin embargo, se observan diferentes grados de percepción según el nivel de educación, lugar de residencia, estado civil, situación económica durante la niñez, situación económica actual y satisfacción general con la vida.

En general, se confirman distinciones de género, educación y satisfacción general con la vida que se explican en el ocio como fuente de felicidad, sin embargo, no resultaron significativos el lugar de residencia, estado civil, situación económica en la niñez y si el adulto mayor es el responsable principal de los ingresos de su hogar.

Finalmente, se determina que la probabilidad de una alta satisfacción con la vida disminuye en la medida en que los AM no consideren importante la familia como fuente de felicidad. Por otra parte la probabilidad de alta satisfacción con la vida aumenta cuando la persona no considera importante el ocio como fuente de felicidad.

\section{Referencias}

Alcalde, I. y Laspeñas, M. L. (2005). Ocio en los mayores: calidad de vida. En J. Giró, Envejecimiento, salud y dependencia (pp. 43-62). Universidad de La Rioja, Servicio de Publicaciones. Aristegui, I. y Silvestre, M. (2012). El ocio como valor en la sociedad actual. Arbor, 188(754), 283aǰ̌a Žganec, A Merkaš, -Ziganec, A., Merkaš, M. y Sverko, I. (2010). Quality of Life and Leisure Activities: How do Leisure Activities Contribute to Subjective Well-Being? Social Indicators Research, 102(1), 81-91. https://doi.org/10.1007/s11205-010-9724-2 Cronbach, L. J. (1982). Designing evaluation of educational and social program. SanFrancisco: Jossey Bass. Cuenca, M. (2007). Ocio y mayores: reflexiones prácticas desde la universidad. Presentado en V Jornadas Municipales "Familia y Comunidd", Agüimes (Gran Canaria): Insituto de Ocio. Universidad de Deusto. Cumming, E. y Henry, W. E. (1961). Growing old, the process of disengagement. Basic Books. Diener E. Emmons, R.A., Larsen, R.J., And Griffin, S. (1985). The Satisfaction with Life Scale. Journal of Personality Assessment. 1985; 49: 71-75. En: Oishi, S. 2000

Diener, E. y Lucas, R. E. (200o). Explaining Differences in Societal Levels of Happiness: Relative Standards, Need Fulfillment, Culture, and Evaluation Theory. Journal of Happiness Studies, 1(1), 41-78. https://doi.org/10.1023/A:1010076127199 Diener, E. y Suh, E. (1997). Measuring quality of life: Economic, social, and subjective indicators. Social indicators research,

iener, E Suh 1 1 . well-being: Three decades of progress. Psychological bulletin, 125(2). Recuperado a partir de https://ssrn.com/abstract=2199216 Eyssautier M. Metodología de la Investigación. Quinta Edición. Editorial Thomson. México. 316 pp. (2006) Fernández-Ballesteros, R., Zamarrón, M. D. y Ruíz, M. A. (2001). The contribution of socio-demographic and psychosocial factors to life satisfaction. Ageing \&amp; Society, 21(1), 25-43. $\quad$ https://doi.org/10.1017/So144686Xo1008078 , y McKenna, K. (1999). Influences on Leisure and Life Satisfaction of Elderly People. Physical \& Occupational Therapy In Geriatrics, 15(4), 1-16. https://doi.org/10.1080/J148V15no4_01 Havighurst, R. J. (1961). Successful aging. Gerontolist, 1, 8-13. $\begin{array}{llll}\text { Havighurst, R. J. (1961). Successful aging. Gerontolist, 1, 8-13. } & \text { (1953). } & \text { Older } \\ \text { Havighurst, } & \text { R. y Albrecht, R. (195) }\end{array}$ People. New York , NY: Longmans, Green. Heo, J., Stebbins, R. A., Kim, J. y' Lee, I. (2013). Serious leisure, life satisfaction, and health of older adults. Leisure Sciences, 35(1), 16-32. https://doi.org/10.1080/01490400.2013.739871 Hernández R, Fernández C, Baptista P. (2006). Metodología de la Investigación. Cuarta Edición. Editorial McGraw Hill InteramericanaEditores S A DeCV. MéxicoD F. México 850 págs.

Herrera A y Cuzmán A. (2012). Reflexiones sobre calidad de vida dignidad y envejecimiento. Revista Médica Clínica Las Condes, 23(1), 65-76. https://doi.org/10.1016/So716-8640(12)70275-4 Hersch, G. (1991). Leisure and Aging. Physical \& Occupational Therapy In Geriatrics, 9(2), 55-78. https://doi.org/10.1080/J148vo9no2_04 
INEC. (2016). Instituto Nacional de Estadística y Censos. Recuperado 3 de agosto de 2016, a partir de http://www.ecuadorencifras.gob.ec efrancois, R., Leclerc, G. y Poulin, N. (1997). Predictors of activity involvement among older adults. Activities, Adaptation \& Aging, 22(4), 15-29. Lemon, B. W., Bengtson, V. L. y Peterson, J. A. (1972). An Exploration of the Activity Theory of Aging: Activity Types and Life Satisfaction Among In-movers to a Retirement Community. Journal of

Leung, L. y Lee, P. (2005). Multiple determinants of life quality: the roles of Internet activities, use of new media, social the roles of Internet activities, use of new media, social
support, and leisure activities. Telematics and Informatics, 22(3), 161-180. https://doi.org/10.1016/j.tele.2004.04.003 Likert R. (1932). A Technique for the Measurement of Attitudes, Archives of Psychology,;140,1-55

Lloyd, K. M. y Auld, C. J.(2002). The Roleof Leisurein Determining Quality of Life: Issues of Content and Measurement. Social Indicators Research, 57(1), 43-71. https://doi.org/10.1023/A:1013879518210

Lyubomirsky, S. y Lepper, H. S. (1999). A measure of subjective happiness: Preliminary reliability and construct happiness: Preliminary reliability and construct
validation. Social indicators research, 46(2), 137-155. Maslow, Abraham H. (1991). Motivación y personalidad. Edición ilustrada, Ediciones Díaz de Santos, ISBN: $\quad 8487189849, \quad 9788487189845, \quad 496$ págs.

Michalkó, G. y Rátz, T. (2010). Measurement of Tourismoriented Aspects of Quality of Life. Journal of Tourism Challenges and Trends, III(2), 35-50.

Moyano, E. y Ramos, N. (2007). Bienestar subjetivo: midiendo satisfacción vital, felicidad y salud en población chilena de la Región Maule. Universum (Talca), 22(2), 177193. https://doi.org/10.4067/So718-23762007000200012

Munné, F. y Codina, N. (1996). Psicología Social del ocio y el tiempo libre. En J. L. Âlvaro, A. Garrido, \& J. R. Torregosa, Psicología Social Aplicada. Madrid: Mc Graw Hill. Narro, S., Aleixandre, M. y Meléndez, J. C. (1994). Actividad y tiempolibre en la tercera edad. Revista de Psicología de la Educación, (14), 5-24.

Newman, D. B., Tay, L. y Diener, E. (2013). Leisure and Subjective Well-Being: A Model of Psychological Mechanisms as Mediating Factors. Journal of Happiness Studies, 15(3), 555-578. https://doi.org/10.1007/s10902-013-9435-X

Newman, D. B., Tay, L., \& Diener, E. (2013). Leisure and Subjective Well-Being: A Model of Psychological Mechanisms as Mediating Factors. Journal of Happiness Studies, 15(3), 555-578. https://doi.org/10.1007/s10902-013-9435-X Nimrod, G. (2007). Retirees' Leisure: Activities, Benefits, and their Contribution to Life Satisfaction. Leisure Studies, 26(1), 65-80. https://doi.org/10.108o/02614360500333937
OMS (2016) Organización Mundial de la Salud. Recuperado 3 de agosto de 2016, a partir de http:// www.who.int/mediacentre/factsheets/fs 404 / es/

OMS. Organización Mundial de la Salud. (2015). Informe Mundial Sobre el Envejecimiento y la Salud (p. 266).

Parker, M. (1996). The Relationship Between Time Spent by older Adults in Leisure Activities and Life Satisfaction. Physical \& Occupational Therapy In Geriatrics, Physical \& Occupational Therapy In Geriatrics,
14(3). Pérez López, C. (2005). Técnicas Estadísticascon SPSS12. Primera Edición. Editorial Pearson Educación, S.A., Madrid, España. 824 Pp. 2005.

Robinson, J. P. y Martin, S. (2008). What Do Happy People Do? Social Indicators Research, 89(3), 565571. $\quad$ https://doi.org/10.1007/s11205-008-9296-6

Rodríguez, A., Látková, P. y Sun, Y.-Y. (2007). The relationship between leisure and life satisfaction: application of activity and need theory. Social Indicators Research, 86(1), 163. https://doi.org/10.1007/s11205-007-9101-y

Schnettler, B., Miranda, H., Sepúlveda, J, Orellana, L., Denegri, M., Mora, M. y Lobos, G. (2014). Variables que influyen M., Mora, M. y Lobos, G. (2014). Variables que influyen
en la satisfacción con la vida de personas de distinto nivel socioeconómico en el sur de Chile. Suma Psicológica, 21(1), 54-62. https://doi.org/10.1016/So121-4381(14)70007-4

Theurer, K. y Wister, A. (2010). Altruistic behaviour and social capital as predictors of well-being among older Canadians. Ageing and Society, 30(1), 157.

Tinsley, H. y Eldredge, B. (1995). Psychological benefits of leisure participation: A taxonomy of leisure activities based on their need-gratifying properties. Journal of Counseling Psychology, 42(2), 123-132. https://doi.org/10.1037/0022-0167.42.2.123

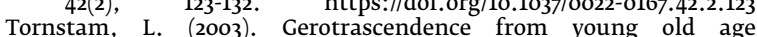
to old old age. Online publication from The Social Gerontology Group, Uppsala.URL: http://www.soc.uu.se/ publications/fulltext/gtransoldold.pdf $\odot$ Lars Tornstam.

Urzúa, M., Loyola, M., Navarrete, M. y Valenzuela, F. (2014). El efecto de valorar la importancia atribuida a cada área de la vida en el auto reporte de la calidad de vida en adultos mayores. Revista argentina de clínica psicológica, 23(1), 41-50.

Walpole, R. E., Myers, R. H., Myers, S. L. y Ye, K. (2007). Probabilidad $\mathrm{y}$ estadística para ingeniería y ciencias (No. TA430. P76 2012.). Pearson Educación.

Wendel-Vos, G. C. W. Schuit, A. J., Tijhuis, M. a. R. y Kromhout, D. (2004). Leisure time physical activity and healthrelated quality of life: Cross-sectional and longitudinal associations. Quality of Life Research, 13(3), 667-677. https://doi.org/10.1023/B:QURE.0oooo21313.51397.33 